\title{
WAVELET-BASED DETECTION OF FREQUENCY HOPPING SIGNALS
}

\author{
Monique P. Fargues, Howard F. Overdyk, and Ralph Hippenstiel \\ Department of Electrical and Computer Engineering \\ Naval Postgraduate School \\ Monterey, CA 93943-5121, USA \\ E-mail: fargues(a)ece.nps.navy.mil
}

\begin{abstract}
We investigate the application of wavelet transforms in the detection and estimation of spread spectrum frequency hopping signals. The technique developed in this work makes only two basic assumptions of a minimum hopping time and a minimum frequency hopping differential. The approach is based on the phase information of the temporal correlation function and the resulting discrete wavelet transform of the phase information is used to estimate the hopping time of frequency hopping signals. Results show the proposed scheme is robust to additive white noise degradations for SNR levels of $3 \mathrm{~dB}$ and above.
\end{abstract}

Keywords: Wavelets, detection, communication.

\section{INTRODUCTION}

Spread spectrum communications schemes have received ever increasing attention over the past two decades as numerous civilian applications have joined the military applications [1]. With this increased use of spread-spectrum communications, naturally, came the question of interception. This question has, therefore, given rise to a considerable amount of research over the past decade focused on the detection and estimation of spread-spectrum communications signals $[2-6,8]$. Two main assumptions typically found in the literature are that the hop timing is constant and known, and that the hopping frequencies are selected from a known class of candidate frequencies. Even when the hop timing is not assumed known, it is still usually assumed constant [3]. These assumptions generally restrict the detection and estimation schemes to frequency hopping $(\mathrm{FH})$, one of the more popular spread-spectrum communications techniques.

A spread-spectrum communications signal consists of both long duration relatively low frequency components ("large" scale) in the carrier frequencies and short duration high frequency components ("small" scale) in the transitional hops. As mentioned above, wavelet analysis is, in theory, well suited for analyzing signals of this type, and had been applied on raw communication signals to detect and classify various schemes with mixed results $[4,8]$.
The primary goal of this paper is to provide a new approach for the detection and estimation of frequency hopping signals which makes none of the restrictive assumptions listed above. By not making such restrictive assumptions, it is hoped that a secondary goal of wider application to the detection and estimation of other spreadspectrum communications techniques, such as direct sequencing, time hopping and hybrids of the three, etc.. [1], can be obtained.

Section 2 defines the temporal correlation function used as the backbone of the analyzing scheme. Section 3 introduces the preprocessing tools used to increase the robustness of the analyzing to noise. Next, Section 4 briefly explains how wavelet analysis fits in our procedure. Section 5 presents the detection scheme developed. Section 6 presents the overall detection algorithm and simulation results. Finally, Section 7 provides conclusions and proposed extensions.

\section{TEMPORAL CORRELATION FUNCTION}

The temporal correlation function (TCF) of a signal $x(k)$ is defined as:

$$
T C F_{x}(k, \tau)=x(k+\tau) x^{*}(k-\tau),
$$

where $\mathrm{k}$ is the absolute center time and $\tau$ is the lag time, expressed in number of samples. Consider the following analytical frequency hopping signal $x(k)$ given by:

$$
x(k)=e^{2 \pi i f_{1} k}\left[u(k)-u\left(k-T_{h o p}\right)\right]+e^{2 \pi i f_{2} k}\left[u\left(k-T_{h o p}+1\right)-u(k-T],\right.
$$

for $0<\mathrm{k}<\mathrm{T}$, where $T_{\text {hop }}$ is the time of the hop (or change in frequency) from $f_{1}$ to $f$, and where $u(k)$ is the unit step function. The resulting TCF function is defined as:

$$
\begin{aligned}
T C F(k, \tau)= & e^{2 \pi /\left(2 f_{1}\right) \tau} D_{1}(k, \tau)+e^{2 \pi j\left(2 f_{2}\right) \tau} D_{2}(k, \tau)+ \\
& e^{j 2 \pi\left[\left(f_{2}-f_{1}\right) k+f_{1}+f_{2}\right) \tau} D_{12}(k, \tau) \\
-T C F_{1}(k, \tau)+T C F_{2}(k, \tau)+T C F_{12}(k, \tau), &
\end{aligned}
$$


where $\mathrm{TCF}_{1}(\mathrm{k}, \tau), \mathrm{TCF}_{2}(k, \tau)$, and $\mathrm{CCF}_{12}(k, \tau)$ represent the $1^{\text {st }}, 2^{\text {nd }}$, and $3^{\text {rd }}$ non overlapping terms contained in the TCF expression. Note that computing the ICF of the real frequency hopping signal has drawbacks as additional "crossterms" are present in the resulting expression, making the frequency identification process more complex [9]. Thus, we consider the analytical frequency hopping signal only in the rest of the study.

Figure 1.a presents the phase plot of an analytical frequency hopping signal $\mathrm{x}(\mathrm{k})$ for some arbitrary $f_{l}$ and $f_{2}$, hoping time $\mathrm{T}_{\text {hop }}=208$, and for for positive $\tau$ values. The combinations of the different shifted versions of the unit step functions force the TCF to take on non-zero values only within the overall triangular within the regions shown in Fig. 1.a. Note that:

1) $\mathrm{TCF}_{1}(\mathrm{k}, \tau)$ is a function of $f_{i}$ and $\tau$ only. The second term, $\mathrm{TCF}_{2}(\mathrm{k}, \tau)$, is a function of $f_{2}$ and $\tau$ only, while the last term, $\operatorname{TCF}_{12}(\mathrm{k}, \tau)$, is a function of $f_{l}, f_{2}, \mathrm{k}$, and $\tau$

2) The frequency hopping time $T_{\text {hop }}$ is located where the region covered by $\mathrm{TCF}_{1}(\mathrm{k}, \tau)$ ends and the region covered by $\mathrm{TCF}_{2}(k, \tau)$ begins.

3) For a given value of $\tau$, the terms within the triangular regions (i.e., the regions where $\operatorname{TCF}_{1}(k, \tau)$ and $\mathrm{TCF}_{2}(\mathrm{k}, \tau)$ are defined) are constant, although at different levels, while the phase behavior within the "cross-terms" region, $\mathrm{TCF}_{12}(\mathrm{k}, \tau)$ is linear. This fact is further illustrated in Fig. 1.b which plots the unwrapped phase of the TCF function for the value $\tau=30$. It is important to realize, however, that the phase values over these three regions is a function of $f_{1}$ and $f_{2}$ and, therefore, not predictable without knowing $f_{1}$ and $f_{2}$, which in general we do not. Nevertheless, for any given value of the lag, $\tau<T_{\text {hop }}$, this region of cross-terms is centered on the hop time, $T_{\text {hop }}$, another fact which is exploited.

\section{PREPROCESSING STEPS}

The main idea behind the proposed scheme is to take advantage of the TCF phase behavior along the time axis $\mathrm{k}$ (i.e., for fixed values of $\tau$ ). As shown earlier the unwrapped TCF phase along the time axis is constant prior and after the frequency jump, while it is linear in a region centered around the frequency hopping time, resulting in a constant-ramp-constant phase behavior along the time axis $\mathrm{k}$, as illustrated in Fig. 1.b. Differentiating such phase leads to a pulse centered around the hopping time, as shown in Fig. 1.c. Detecting the edges of the pulse is then all what is needed to identify the hopping time, as it can then be estimated as the midpoint between the two edges. Therefore, the hopping time detection problem can be viewed as an edge detection problem, which the wavelet transform is well-matched to address
However, additive noise contained in the communication signal results in phase noise which degrades the quality of the resulting pulse. Furthermore, the differentiating step increases the effects due to noise. Thus, we apply median filtering before and after the differentiating step to minimize these phase noise effects. The main advantage behind this filter is that it preserves the ramp and step behavior and eliminates outliers. The length of the median filter operation was selected in order to preserve the step discontinuities present in $\operatorname{TCF}(k, \tau)$ for fixed $\tau[9]$.

\section{WAVELET TRANSFORM}

Edge detection is an important problem in numerous applications ranging from image processing to transient detection, and wavelet transforms have been used extensively for detecting discontinuities in a given signal or its derivatives. Recall that wavelets may be used to detect discontinuities in a signal or its derivatives, if the chosen wavelet function is able to represent the highest order derivative present in the signal function, as any wavelet with, at least $\mathrm{p}$ vanishing moments, can be used to detect a discontinuity in the $(\mathrm{p}-1)^{\mathrm{st}}$ derivative [7]. For our problem, we are interested in identifying pulse edges, i.e., a signal discontinuity, and the Haar wavelet is sufficient for the task.

The presence of noise makes identification of discontinuities more complicated. In such a case, the averaging of several scales can enhance the wavelet's ability to detect discontinuities in noise. The basic idea is that for true discontinuities, the spikes will line up, while those associated to noise will not [7]. As a result, we averaged the first two scales in our simulation to improve the robustness of the detection scheme to noise degradations.

Fig. 2a plots the result obtained by averaging the first two scales of the DWT applied to the unwrapped phase of the TCF expression for an analytical frequency hopping $(\mathrm{FH})$ signal. The $\mathrm{FH}$ function has frequencies on either side of the hop, located at time sample 208, $f_{1}=$ $6.250 \mathrm{MHZ}$ and $f_{2}=22.727 \mathrm{MHZ}$. The SNR level is equal to $10 \mathrm{~dB}$. Fig. $2 \mathrm{~b}$ illustrates the resulting wavelet transform of the preprocessed phase of the TCF obtained at lag $\tau=30$. Fig. $2 b$ shows that the wavelet transform clearly detect the location of the pulse ends, as expected. Note that the width between each spikes obtained from taking the wavelet transform of the TCF phase along the time axis $k$ (i.e., for fixed lag time $\tau$ ) increases as $\tau$ increases. The next step sums all the values which represent the edges of the crossterms in the TCF in $45^{\circ}$ and $135^{\circ}$ directions so that they reinforce each other, as illustrated in Fig. 3.b. Fig. 3c shows the "detection vector" obtained from this summation. 
Further details may be found in Overdyk [9]. Note that the resulting spike is located at the hopping time $\mathrm{T}_{\text {hop }}=208$.

\section{DETECTION SCHEME}

Once the detection vector has been formed a decision must be made as to whether or not a hop has occurred within the frame. Experiments suggested that the variance of the detection vector would be the best indicator of whether or not a hop had occurred. As a result, the threshold, $T_{\text {treshoch }}$ is chosen as a multiple, $k$, of the variance of the detection vector when no hop has occurred within the frame. The threshold determination was also guided by the fact that the cost associated with the probability of a missed detection, $P_{m}=\left[1\right.$ - probability of detection $\left.\left(P_{D}\right)\right]$ far outweighs the cost associated with the probability of a false alarm, $P_{F A}$, as the hopping time estimation is only the first step in a complete frequency hopping signal detection scheme.

Note that once the hopping times are estimated, the signal frequencies need to be extracted to demodulate the actual message. This step can easily be done by applying frequency analysis to the estimated hopping intervals. Thus, in the case of false alarms, frequency analysis would show the same frequencies in two, or more, consecutive hopping intervals, resulting in no message degradation. However, a missed hopping time will result in degradations in the frequency estimation step, and errors in the decoded message. Receiver operating characteristics (ROC) curves were generated for each of the six SNR levels and an appropriate threshold chosen for each [9].

\section{DETECTION ALGORITHM AND RESULTS}

As stated earlier, it was desired to make as few assumptions as possible on the nature of the frequency hopping signal. With this goal mind the assumptions were limited to three:

1. Known spread spectrum frequency range. This range was assumed to be limited from $1 \mathrm{MHZ}$ to $24 \mathrm{MHZ}$ in the simulations conducted,

2. Known minimum hopping time, $\mathrm{T}_{\text {hop_min. }}$ This parameter was chosen to be equal to 256 sample points in our simulations. At a sampling rate of $50 \mathrm{MHZ}$, this translates into a minimum hopping time of $5.12 \mu \mathrm{s}$,

3. Minimum frequency differential, $\Delta f$, for frequency hops. This parameter was chosen to be $1 \mathrm{Khz}$ in our simulations.

\section{Detection Algorithm}

Using the tools described above, the algorithm steps for the detection and estimation of frequency hopping signals in noise can now be enumerated as follows:

1. Transform the real signal into an analytic signal.

2. Segment data into frames of length less than or equal to the minimum hopping time, $T_{\text {hop_mir }}$ This assumption ensures that, at most, one hop will be present in the processing frame.

3. Compute the temporal correlation function on each frame.

4. Extract the TCF phase information and unwrap the phase along the time axis $\mathrm{k}$.

5. Apply a median filter to the phase of the TCF along the time axis $\mathrm{k}$, of length 5 . This step is done to reduce the noise effects prior to differentiating, since differentiating accentuates these effects due to noise.

6. Differentiate the phase information along the time axis $\mathrm{k}$. This step changes the unwrapped phase of the TCF from a ramp function to a pseudo-pulse.

7. Apply another median filter along the time axis $\mathrm{k}$ of length 25 . The length of 25 has proven to work well with the $T_{\text {hop min }}$ chosen for our simulations [9]. This step is done to again remove the effects due to noise which were accentuated by the differentiation operation in step 6 .

8. Calculate DWTs along the time axis $k$ (i.e. for each lag, $\tau$, of the TCF) using the Haar wavelet. This step is done to detect the discontinuities at the edges of the cross-terms region

9. Average the wavelet coefficients of the first two scales of the DWT

10. Perform a $45^{\circ} / 135^{\circ}$ summation across all values of lag, $\tau$, to obtain a detection vector which has time as its index. 11. Threshold the data in the resulting detection vector obtained in step 10. When detected above the threshold, the maximum peak value time index represents the estimated hopping time.

\section{Simulation results}

Simulations were conducted to test the effectiveness of the detection and estimation algorithm given above. Five hundred trial experiments were conducted in six different signal to noise ratios (SNR) between -3 to $15 \mathrm{~dB}$. The basic idea behind the experiments was to simulate signals that had already been segmented, as specified in steps 1 and 2 of the algorithm. The problem then becomes to determine: a) whether or not a frequency hop exists within the given frame; b) to estimate the hopping time when a frequency hop is detected

Communication signals were generated by choosing random hopping time, $\mathrm{T}_{\text {hop }}$, and hopping frequencies $f_{1} f_{2}$ selected randomly in a predefined range. The resulting signal is a signal with, at most, one hop which can be from any frequency, $f_{l}$, to any hopping 
frequency, such that $1 \mathrm{MHZ} \leq f_{1} f_{2} \leq 24 \mathrm{MHZ}$.

Table 1 shows the probability of detection, $P_{D}$, the probability of false alarm, $\mathrm{P}_{\mathrm{FA}}$, and the percentage of emors in classification for the selected threshold, $T_{\text {threshold }}$, at each of the six SNRs considered. Note the entries under the column labeled " $k$ " represents a multiple of the variance of the detection vector generated from a "no hop" frame for each respective SNR level. The column labeled "\% Error" shows the percentage of misclassifications (i.e., the percentage of false alarms plus misses). Note, also, that the a low probability of false alarm was sacrificed for higher probabilities of detection for reasons discussed earlier. For example, the entries in the row for $\mathrm{SNR}=3 \mathrm{~dB}$ show that if a $11.4 \%$ misclassification rate and a $P_{F A}=0.1961$ can be tolerated, then we can expect to detect $89.53 \%$ of the hops in a given frequency hopping signal.

Simulation results are shown in Table 2 . The column labeled "Avg. Enror" gives the average error obtained at each SNR level. For example, at the SNR level of $3 \mathrm{~dB}$, out of all the hops which were detected, the average distance from the true hopping time was 10.48 sample points (i.e., $4.1 \%$ of the minimum hopping time) Columns with numeric headings indicate the hop detection probability within a given percentage of $T_{\text {hop_mir }}$ For example, at the SNR level of $3 \mathrm{~dB}$, the column labeled " $1 \%$ " indicates that $36 \%$ of all detected hops were located within $1 \%$ of $T_{\text {hop mir }}$ or within 2 points of the true hop time, $T_{\text {hop }}$. Similarly, $72 \%$ of all detected hops were located within $5 \%$ of $T_{\text {hop } \min }$ or within 12 points of the true hop time, $T_{h o p}$

\section{CONCLUSIONS}

This work considered the application of temporal correlation functions and wavelet analysis to the detection and estimation of frequency hopping signals in additive white Gaussian noise. The algorithm developed has only two restrictive assumptions: 1) a minimum hopping time; 2) a minimum frequency differential. Thus, it can find applications where the minimum hopping time is not held constant; i.e., in time hopping signals and hybrid techniques involving either frequency hopping or time hopping. We showed how the detection problem can be formulated as an edge detection problem, and how wavelet analysis can be used in the hopping time detection problem. Third, we introduced preprocessing techniques designed to improve the robustness of the detection and estimation scheme in noisy environments. Results show that the scheme is robust to noise degradations down to $3 \mathrm{~dB}$.

Although the algorithm developed succeeded in meeting its goals, improvements can potentially be obtained by taking advantages of the specific twodimensional structure of the hopping pattern. Thus, a possible extension involves considering the problem as an image processing or pattern recognition problem, due to the specific triangular pattern generated by the TCF of frequency hopping signals. As a result, applying more sophisticated two-dimensional edge detection schemes may improve the robustness of the detection and estimation scheme. Such extensions are presently under study.

\section{REFERENCES}

[1] R. Peterson, R. Ziemer, \& D. Borth, Introduction to spread spectrum communications, Prentice Hall, 1995.

[2] N. Beaulieu, W. Hopkins, \& P. McLane, "Interception of frequency-hopped spread-spectrum signals," IEEE J. Select. Areas Commun., Vol. 8, No. 5, pp. 853-870, June 1990.

[3] L. Aydin \& A. Polydoros, "Hop-timing estimation for FH signals using a coarsely channelized receiver," IEEE Trans. Commun., Vol. 44, No. 4, April 1996.

[4] K. Ho, W. Prokopiw, \& Y. Chan, "Modulation identification by the wavelet transform," Proceedings of MILCOM 95, Nov. 1995.

[5] M. Simon, U. Cheng, L. Aydin, A. Polydoros, and B. Levitt, "Hop timing estimation for noncoherent frequency hopped M-FSK intercept receivers, IEEE Trans. Commun., Vol. 43, No. 2/3/4, pp. 1144-1154, Feb.March/Ap. 1995

[6] C. Chung \& A. Polydoros, "Parameter estimation of random $\mathrm{FH}$ signals using autocorrelation techniques, IEEE Trans. Commun. Vol. 40, No. 1, pp. 149-159, Jan. 1992

[7] G. Strang and T. Nguyen, Wavelets and filter banks, Wellesley, 1996

[8] D. Cochran, Application of wavelet transform techniques to spread spectrum demodulation and jamming, Tech. Report, Arizona State University, Feb. 1993.

[9] Howard F. Overdyk, Detection and estimation of frequency hopping signals using wavelet transforms, MSEE Thesis, Naval Postgraduate School, Sept. 1997. 


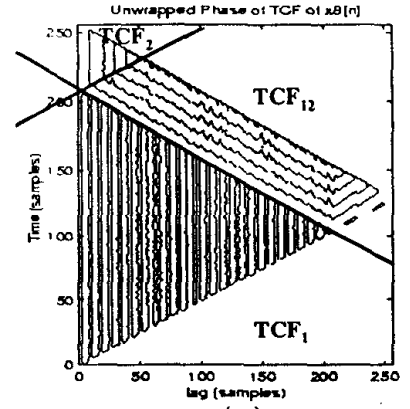

(a)

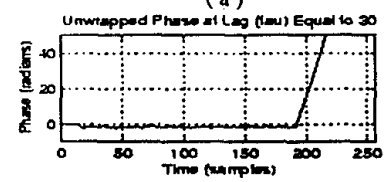

(b)

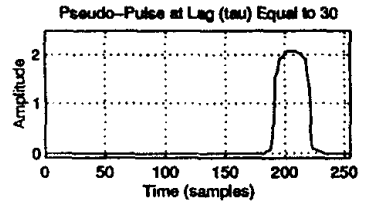

(c)

Figure 1: The unwrapped phase information of (a) the TCF, ( $b$ ) for $\tau=30$, and (c) after differentiating and median filtering for $\tau=30$.

\begin{tabular}{|l||l|l|l|l|}
\hline SNR & $k$ & $P_{d}$ & $P_{\mathrm{fa}}$ & $\%$ Error \\
\hline $15 \mathrm{~dB}$ & 140 & 1 & 0 & 0.0 \\
\hline $10 \mathrm{~dB}$ & 30 & 0.9866 & 0.0196 & 1.4 \\
\hline $6 \mathrm{~dB}$ & 15 & 0.9844 & 0.1569 & 3.0 \\
\hline $3 \mathrm{~dB}$ & 11 & 0.8953 & 0.1961 & 11.4 \\
\hline $0 \mathrm{~dB}$ & 1 & 0.8129 & 0.3529 & 20.4 \\
\hline $3 \mathrm{~dB}$ & 3 & 0.6927 & 0.3333 & 31.0 \\
\hline
\end{tabular}

Table 1: Detection statistics of 500 experiments applying the detection and estimation algorithm.
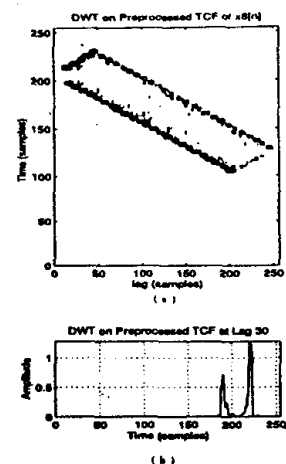

Figure 2: DWT coefficients computed for each value of lag $\tau(a)$ on the preprocessed phase of the $\operatorname{TCF}(t, \tau)$, and for $\tau=30$.

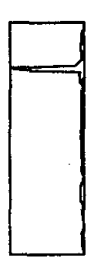

(a)

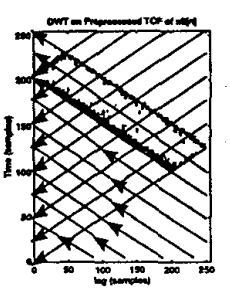

(b)

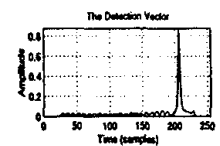

(c)

Figure 3: Detection vector constructed by performing a $45^{\circ} / 135^{\circ}$ summation. ( a ) shows effects of the summation in ( $b$ ) where the arrows indicate the direction of summation. (c) shows a plot of the actual detection vector.

\begin{tabular}{|c||c|c|c|c|c|c|c|c|c|c|c|}
\hline $\begin{array}{c}\text { SNR } \\
(\mathrm{dB})\end{array}$ & $1 \%$ & $5 \%$ & $10 \%$ & $15 \%$ & $20 \%$ & $30 \%$ & $40 \%$ & $50 \%$ & $75 \%$ & $100 \%$ & $\begin{array}{c}\text { Avg. Error } \\
\text { (\# of samples) }\end{array}$ \\
\hline \hline 15 & 0.790 & 0.984 & 0.992 & 0.996 & 0.996 & 0.998 & 1.00 & 1.00 & 1.00 & 1.00 & 2.22 \\
\hline 10 & 0.726 & 0.964 & 0.974 & 0.978 & 0.982 & 0.986 & 0.986 & 0.986 & 0.986 & 0.986 & 2.70 \\
\hline 6 & 0.558 & 0.888 & 0.926 & 0.940 & 0.950 & 0.960 & 0.968 & 0.970 & 0.970 & 0.970 & 5.46 \\
\hline 3 & 0.360 & 0.720 & 0.758 & 0.794 & 0.828 & 0.862 & 0.874 & 0.882 & 0.886 & 0.886 & 10.48 \\
\hline 0 & 0.116 & 0.302 & 0.418 & 0.510 & 0.572 & 0.684 & 0.752 & 0.768 & 0.796 & 0.796 & 28.48 \\
\hline-3 & 0.090 & 0.174 & 0.276 & 0.382 & 0.456 & 0.568 & 0.614 & 0.646 & 0.686 & 0.690 & 30.99 \\
\hline
\end{tabular}

Table - 2: Estimation statistics for the 500 experiments at each SNR level using the detection and estimation algorithm described in this chapter. Columns with numeric headings, show the probability that estimated hops are found within a given distance, expressed in percentage of $T_{\text {hop_min }}$, from true hopping times. 